US Army Corps of Engineers ${ }_{\circledast}$

Engineer Research and

Development Center

Aquatic Plant Control Research Program

\title{
A Novel Laboratory Method for the Detection and Identification of Cyanobacteria Using Hyperspectral Imaging
}

Hyperspectral Imaging for Cyanobacteria Detection

Kaytee Pokrzywinski, Cliff Morgan, Scott Bourne, Molly Reif, June 2021 Kenneth Matheson, and Shea Hammond

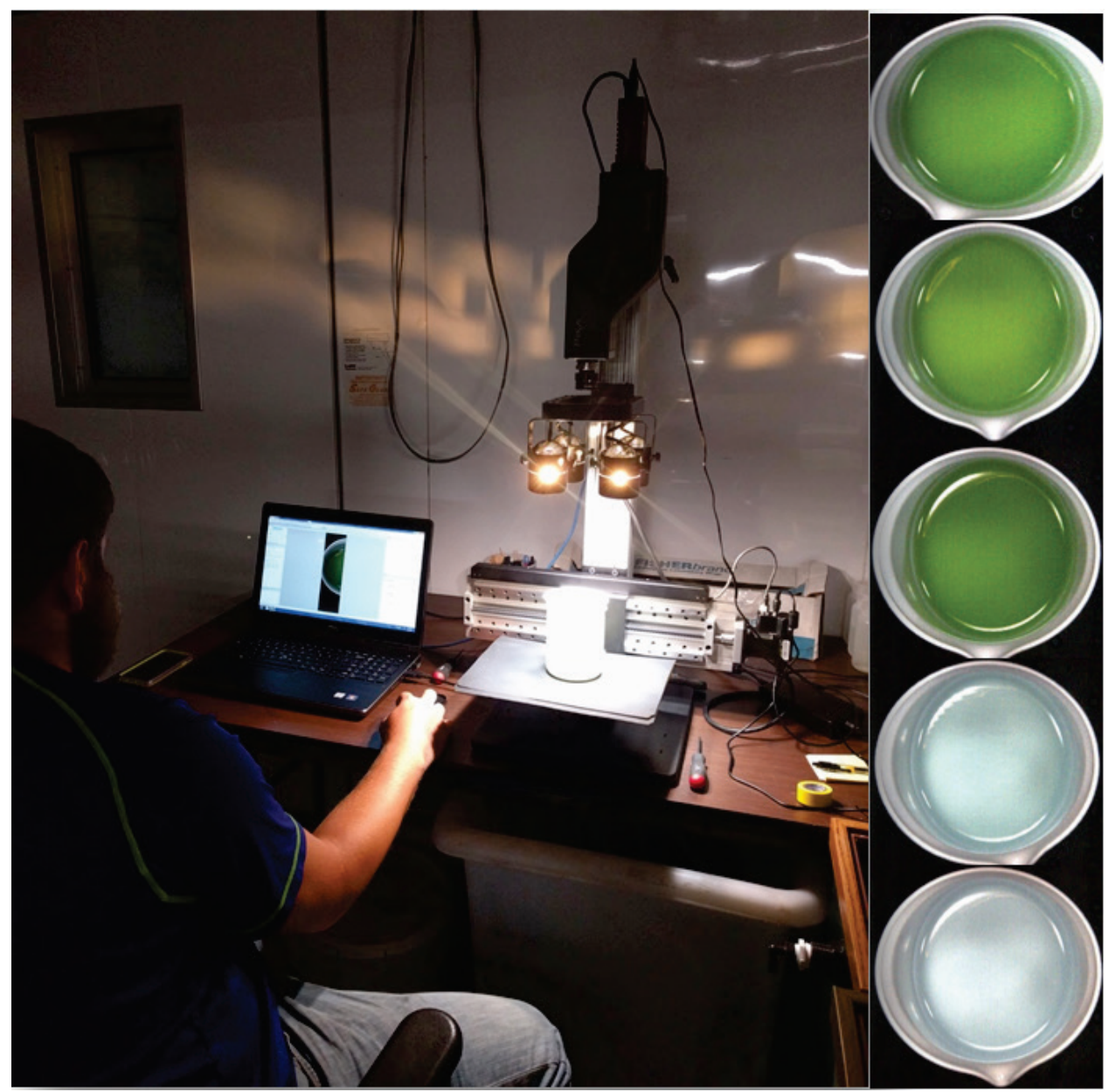


The US Army Engineer Research and Development Center (ERDC) solves the nation's toughest engineering and environmental challenges. ERDC develops innovative solutions in civil and military engineering, geospatial sciences, water resources, and environmental sciences for the Army, the Department of Defense, civilian agencies, and our nation's public good. Find out more at www.erdc.usace.army.mil.

To search for other technical reports published by ERDC, visit the ERDC online library at https://erdclibrary.on.worldcat.org/discovery. 


\section{A Novel Laboratory Method for the Detection and Identification of Cyanobacteria Using Hyperspectral Imaging}

Hyperspectral Imaging for Cyanobacteria Detection

Kaytee Pokrzywinski, Cliff Morgan, Scott Bourne, Molly Reif, Kenneth Matheson, and Shea Hammond

Environmental Laboratory

US Army Engineer Research and Development Center 3909 Halls Ferry Rd

Vicksburg, MS 39180-6199

Final report

Approved for public release; distribution is unlimited.

Prepared for Aquatic Plant Control Research Program US Army Engineer Research and Development Center Vicksburg, MS 39180-6199

Under Funding Account Code U4363405; AMSCO Code 070598 


\section{Abstract}

To assist US Army Corps of Engineers resource managers in monitoring for cyanobacteria bloom events, a laboratory method using hyperspectral imaging has been developed. This method enables the rapid detection of cyanobacteria in large volumes and has the potential to be transitioned to aerial platforms for field deployment. Prior to field data collection, validation of the technology in the laboratory using monocultures was needed. This report describes the development of the detection method using hyperspectral imaging and the stability/reliability of these signatures for identification purposes. Hyperspectral signatures of different cyanobacteria were compared to evaluate spectral deviations between genera to assess the feasibility of using this imaging method in the field. Algorithms were then developed to spectrally deconvolute mixtures of cyanobacteria to determine relative abundances of each species. Last, laboratory cultures of Microcystis aeruginosa and Anabaena sp. were subjected to varying macro (nitrate and phosphate) and micro-nutrient (iron and magnesium) stressors to establish the stability of signatures within each species. Based on the findings, hyperspectral imaging can be a valuable tool for the detection and monitoring of cyanobacteria. However, it should be used with caution and only during stages of active growth for accurate identification and limited interference owing to stress.

DISCLAIMER: The contents of this report are not to be used for advertising, publication, or promotional purposes. Citation of trade names does not constitute an official endorsement or approval of the use of such commercial products. All product names and trademarks cited are the property of their respective owners. The findings of this report are not to be construed as an official Department of the Army position unless so designated by other authorized documents. 


\section{Contents}

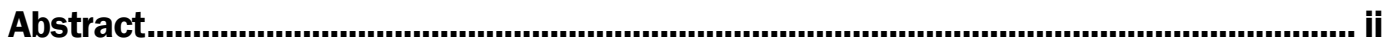

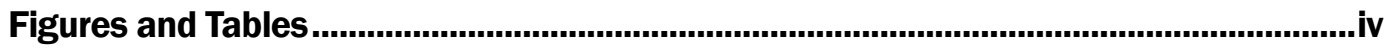

Preface

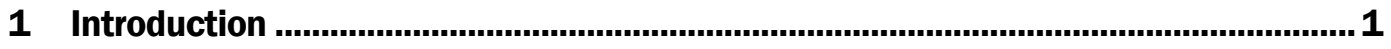

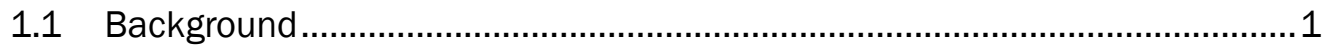

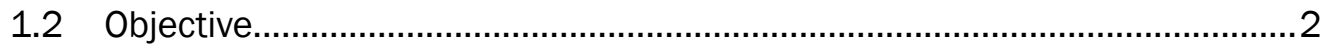

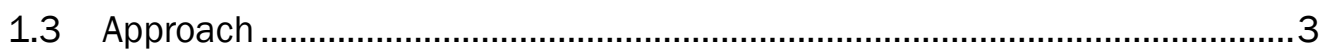

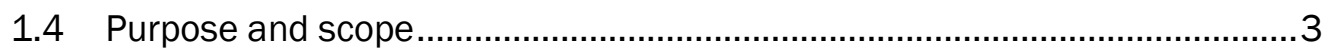

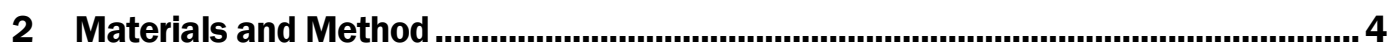

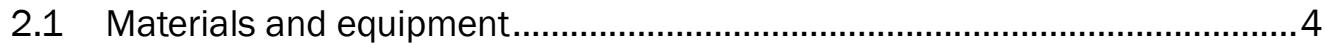

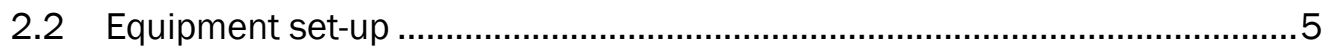

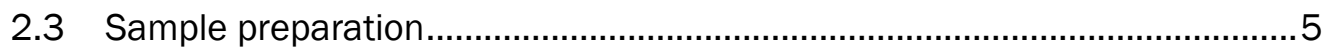

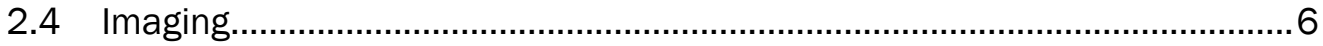

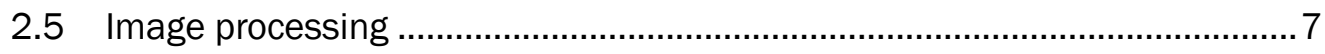

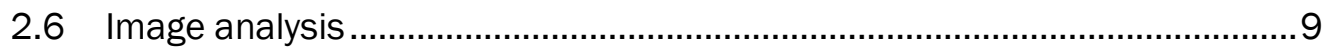

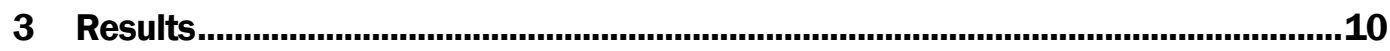

3.1 Light scattering and cyanobacteria concentration ......................................10

3.2 Cyanobacteria spectral comparisons ..................................................... 12

3.3 Separating signatures from co-cultures of cyanobacteria ......................... 12

3.4 Future improvements ......................................................................... 13

4 Conclusions and Future Directions......................................................................14

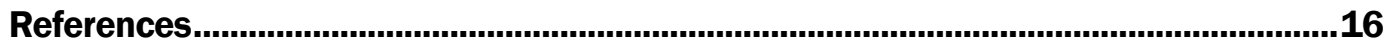

Appendix: R Script for Hyperspectral Data Analysis ......................................................17

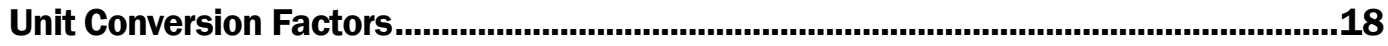

\section{Report Documentation Page}




\section{Figures and Tables}

\section{Figures}

Figure 1. Resonon Pika XC hyperspectral camera setup.................................................. 4

Figure 2. Example sample acquisition.......................................................................... 7

Figure 3. Example region of interest (ROI) (inner green circle).......................................... 8

Figure 4. Image statistics generated in ENVI. ................................................................

Figure 5. Laboratory acquired samples of increasing concentrations of Anabaena (A) and Microcystis (B) cyanobacteria................................................................................

Figure 6. Spectral comparison of different concentrations/dilutions of Anabaena sp. both the raw spectrum $(A)$, instantaneous rate of change $(B)$. .

\section{Tables}

Table 1. Specifications of the Resonon Pika XC hyperspectral camera.................................5 


\section{Preface}

This study was conducted for and funded by the US Army Engineer Research and Development Center (ERDC), Aquatic Plant Control Research Program, under Funding Account Code U4363405; AMSCO Code 070598. Dr. Jennifer Seiter-Moser was the Technical Director, and Mr. Michael Greer, US Army Corps of Engineers, Buffalo District, was the Acting Program Manager.

The work reported herein was performed by the Environmental Processes Branch (EPP), Environmental Processes and Engineering Division (EPED), of the Environmental Laboratory (EL) of the ERDC, Vicksburg, MS.

At the time of publication of this report, Mr. Brandon Lafferty was Chief of EPP; Dr. Todd Steissberg was Acting Chief of EPE; Dr. Jack Davis was the Deputy Director of EL, and Dr. Edmond Russo was the Director.

Appreciation is extended to Mr. Sam Jackson and Dr. Brook Herman for their assistance during peer review.

The Commander of ERDC was COL Teresa A. Schlosser, and the Director was Dr. David W. Pittman. 


\section{Introduction}

\subsection{Background}

Freshwater harmful algal blooms (HABs) have gained increasing attention in recent years. The magnitude of HAB events as well as their frequency and duration in inland waters is not well documented (Brooks et al. 2015), yet multiple lines of evidence indicate that algal blooms have increased in frequency during the past several decades Mehrubeoglu et al. (2014).

The US Army Corps of Engineers manages a significant number of inland waterways that provide a variety of services including flood control, energy production, and navigation as well as associated uses such as recreation, agriculture, and potable water supplies that are covered by the Clean Water Act (Brooks et al. 2015). Early detection of freshwater HABs is important for mitigation strategies and integrated water resource management.

The early detection of freshwater HABs is challenging owing to the size of microscopic organisms and resolution of current technologies, limiting lake-wide, low-level detection of cyanobacteria without the use of point sampling and molecular tools. Tools that allow managers to identify water bodies that are in early bloom stages or prone to toxin release would reduce costs and improve the likelihood of controlling these events. Early detection could also minimize the impact of HABs on municipal water treatment facilities and recreational water through the rapid implementation of response plans.

Current methods for detecting HABs range from field observations and water sampling to airborne and satellite-based imaging (Oppelt et al. 2012; Reif 2011; Reif et al. 2012). However, all of these methods have trade-offs that must be evaluated to select the appropriate detection approach. Fieldbased methods are limited by accessibility and single point samples that may not adequately represent the dynamic nature of larger waterbodies. In contrast, satellite sensors can cover large areas yet face limitations with spatial, spectral, and temporal resolutions that impact detection capabilities and accuracy (Oppelt 2012). Airborne sensors, while providing broader coverage than field observations, can also provide higher spatial resolutions and greater flexibility than satellite sensors yet can be costly when repeat datasets are required for monitoring (Reif 2011). As an 
example, airborne hyperspectral imaging has also been successfully used for species-level classification of submerged aquatic vegetation including macroalgae (Reif et al. 2012). Additionally, remote sensing is routinely used to estimate the concentration and distribution of cyanobacteria (Vincent et al. 2004).

While significant progress has been made in detecting HABs, more work is needed to improve early detection and predict toxic events. Although water quality parameters such as temperature, macronutrient concentration (including nitrogen and phosphorous) and $\mathrm{pH}$ are well studied and routinely monitored, these data have not been comprehensively linked to existing studies of algal growth and toxin release. High concentrations of toxin producing strains do not always correlate to high toxin concentrations, but studies have shown that under stress, toxin levels can increase by $90 \%$ compared to unstressed cells at the same density (Ross et al. 2006). Quantifying the degree of change associated with physical stressors will enable better prediction of toxic bloom events, ultimately allowing managers to make better-informed water management decisions.

\subsection{Objective}

Point sampling efforts alone often miss the early stages of HAB development as they rely on obtaining grab samples from the exact location the bloom initiated, which is often times unknown. Therefore, remote sensing platforms are increasingly being used in combination with, or to help prioritize, point sampling efforts. However, given the current state of remote sensing technology, and subsequent capabilities and inherent limitations (e.g., cloud cover), early signs of HAB detection and prediction of toxin release are likewise limited. Advances in sensor and platform technology continue to push the evolution of new and improved feature detection capabilities, especially those requiring very high spatial (hyperspatial) or spectral (hyperspectral) resolutions to discriminate and resolve subtle characteristics that may otherwise go undetected. Unmanned aircraft system (UAS) technology is one such technological development that shows promise for offering flexible, hyperspatial remote sensing capabilities. To that end, species-specific HAB detection and toxin prediction would benefit from specific research and development utilizing UAS sensors and platforms, in which more detailed feature extraction may be possible. As such, a combination of laboratory and field-based experiments is needed to bridge the gap between current detection and monitoring strategies and, ultimately, to illustrate how UAS technology 
can best be used to address HAB monitoring needs in the midst of a wide variety of remote sensing and field-based approaches.

\subsection{Approach}

This study focused on the development of a laboratory method to rapidly detect cyanobacteria ex situ using hyperspectral imaging. The goal is to establish spectral stability and validate the technology in the laboratory to assist with transitioning the findings to future UAS data collection, processing, and evaluation.

\subsection{Purpose and scope}

This technical report was prepared under the work-unit "Strategies for Early Detection of Harmful Algal Blooms and Predicting Toxin Release: Linking Hyperspectral Imaging to Molecular Techniques" funded by the Aquatic Plant Control Research Program. The purpose of this study was to describe the materials and methodology behind hyperspectral imaging for cyanobacteria monitoring and detection. 


\section{Materials and Method}

\subsection{Materials and equipment}

The benchtop hyperspectral imaging system (Resonon, Inc. 2018) is comprised of the following:

- A Pika XC hyperspectral imaging camera

- Linear translation stage

- Mounting tower

- Lighting assembly

- Software control system.

- $500 \mathrm{~mL}^{*}$ beaker sprayed with reflective paint.

The positions of the imager and lighting assembly are adjustable along the length of the tower. Figure 1 shows the system setup.

Figure 1. Resonon Pika XC hyperspectral camera setup.

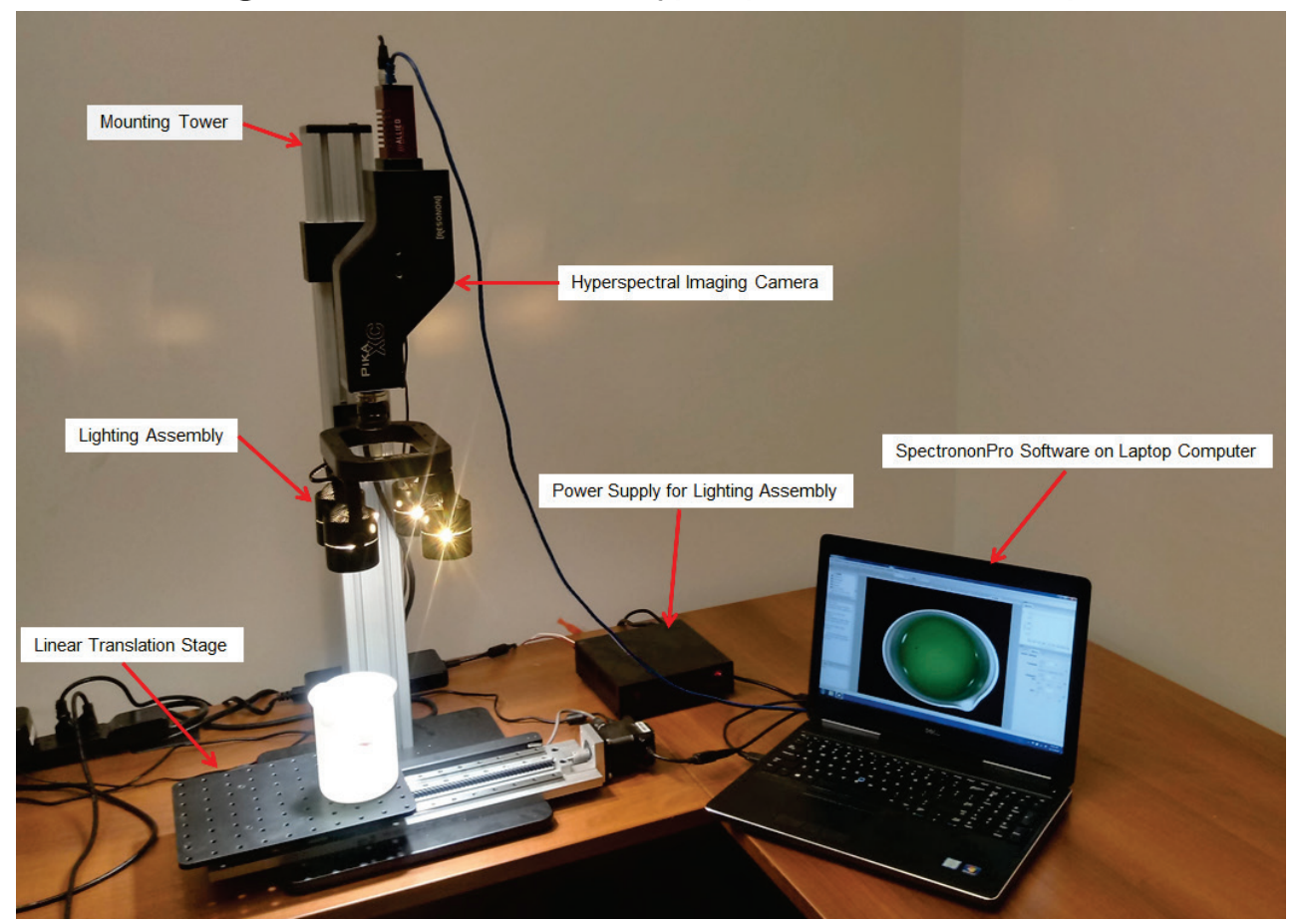

* For a full list of the spelled-out forms of the units of measure used in this document, please refer to US Government Publishing Office Style Manual, 31st ed. (Washington, DC: US Government Publishing Office 2016), 248-52, https://www.govinfo.gov/content/pkg/GPO-STYLEMANUAL-2016/pdf/GPOSTYLEMANUAL-2016.pdf. 


\subsection{Equipment set-up}

The Pika XC high-performance visible-near infrared hyperspectral imager has very high spectral resolution and high spatial quality. The camera and imaging system, which includes the mounting tower, lighting assembly, translation stage, power supply, and camera, are manufactured by Resonon Inc. The lighting system has current-controlled wide-spectrum quartz halogen lights specifically designed for Resonon's hyperspectral imaging cameras. Resonon's hyperspectral imagers are line-scan imagers (also referred to as push-broom imagers). Two-dimensional images were constructed by translating the sample relative to the camera. Table 1 shows the setting, spectral, and spatial resolutions of the imaging system. The camera lens was consistently positioned $12 \mathrm{in.} \mathrm{from} \mathrm{the} \mathrm{top} \mathrm{of} \mathrm{sample} \mathrm{and}$ stage lights were 5 in. from the top of sample.

Table 1. Specifications of the Resonon Pika XC hyperspectral camera.

\begin{tabular}{|l|l|}
\hline Spectral Range & $400-1000 \mathrm{~nm}$ \\
\hline Spectral Resolution & $1.3 \mathrm{~nm}$ \\
\hline Spectral Channels & 447 \\
\hline Max Frame Rate & $165 \mathrm{fps}$ \\
\hline Signal-to-Noise Ratio (peak) & $366(2 \mathrm{x}$ bin) to $518(4 \mathrm{x}$ bin $)$ \\
\hline f/\# & $\mathrm{f} / 2.4$ \\
\hline Pixel Size & $6.86 \mu \mathrm{m}$ \\
\hline
\end{tabular}

\subsection{Sample preparation}

Cultures of various cyanobacteria obtained from the University of Texas Culture Collection (UTEX) were maintained in semi-continuous culture at $23{ }^{\circ} \mathrm{C}$ on a shaker platform $85 \mathrm{rpm}$ in replete BG-11 media. Cultures included Microcystis aeruginosa (UTEX 2385, toxic), Anabaena sp. (UTEX 1448, potentially toxic), Nodularia spumingia (UTEX 2091). For imaging experiments, cultures were started at a cell density of 0.005 optical density $(\mathrm{OD})$ at $650 \mathrm{~nm}\left(\mathrm{OD}_{650}\right)$ and grown under various nutrient conditions for 15 days in $200 \mathrm{~mL}$ of modified BG-11 media. Cultures were monitored for growth and cyanotoxins daily and every 3 days, respectively. For imaging, triplicates were concatenated, and $300 \mathrm{~mL}$ of culture were added to a $500 \mathrm{~mL}$ glass beaker painted with Krylon Ultra Flat White paint Color Code 51502 . The spectral characteristics of this paint were close to $100 \%$ 
reflectance and as such provided minimal signal interference and low signal-to-noise ratios. An amount of $300 \mathrm{~mL}$ of each sample was used as this provided a dense enough sample for viewing without sensor saturation. If needed, cultures were diluted using comparable BG-11 media.

\subsection{Imaging}

After initializing the camera, lights, and computer as instructed by the manufacturer, the SpectrononPro software v2.98 (Hunter et al. 2016) was used to capture a dark reference with the lens cap in place. After which, a white reference was captured by removing the lens cap and imaging a spectralon white reference panel on the camera stage. Once the reference calibration was completed, the frame rate and stage speed were adjusted to obtain square pixels in each image.

During sample acquisition (Figure 2), the exposure parameters were controlled by clicking on the Camera tab in the Tools Panel using the SpectrononPro software. The Frame Rate was equal to the number of images acquired each second and limited the maximum exposure time (Max Exposure Time $=1.0 \div$ Frame Rate). The duration of time required for acquisition of each line image was termed Integration Time (also known as Exposure Time). Gain was a factor, which increases the signal but at the expense of signal-to-noise ratio. In this study, the gain was kept as low as possible (at zero, unless it was necessary to increase it).

Various types of vessels were initially imaged to determine what type of container would provide the lowest signal-to-noise ratio with minimal signal interference. Containers including different size petri dishes (clear plastic), beakers (glass), specimen jars (opaque plastic), and mason jars (glass) were tested both with and without black and white paint provided by the manufacturer. It was determined that a painted (inside) $500 \mathrm{~mL}$ glass beaker containing Krylon Ultra Flat White paint color code 51502 provided the best signatures with the lowest signal-to-noise ratio. 
Figure 2. Example sample acquisition.

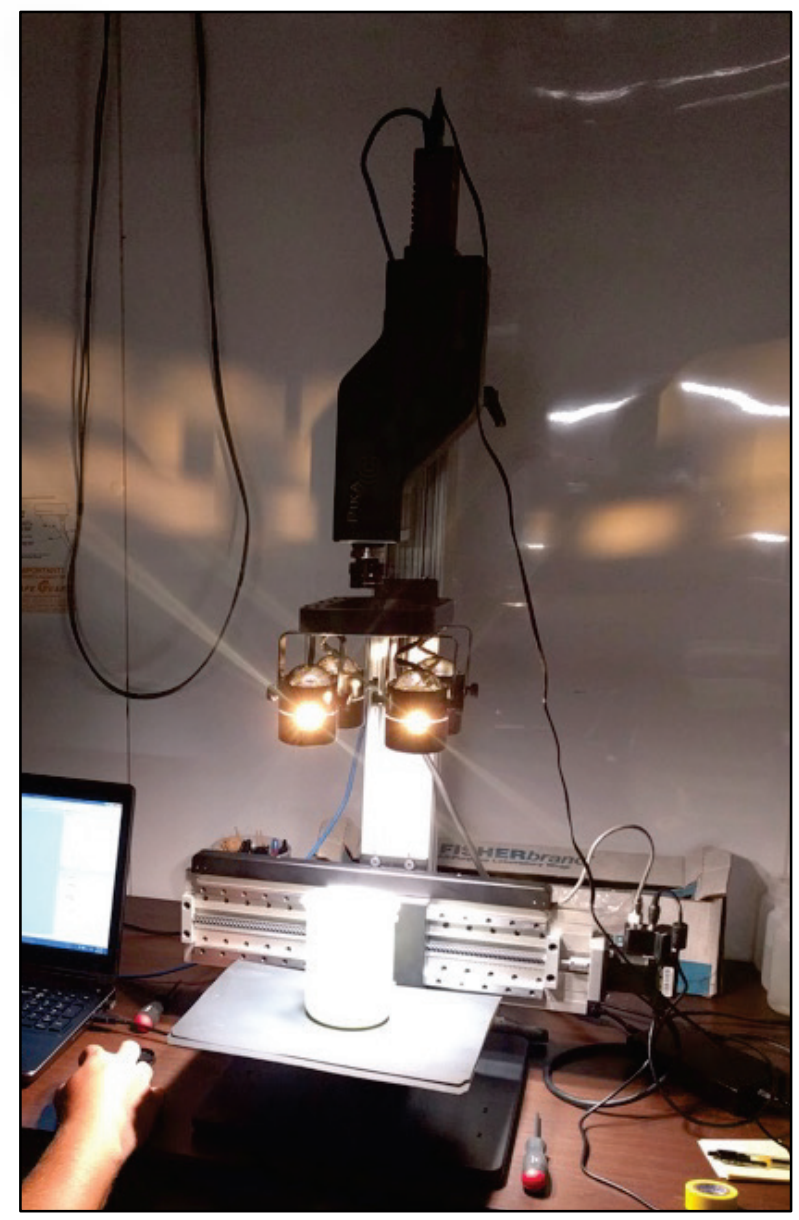

\subsection{Image processing}

This type of hyperspectral imaging is not directly comparable to either conventional absorption or reflection spectroscopy. Rather, it is a combination of the two. Therefore, the intensity of light reflected back to the detector cannot be modeled as a simple linear function. Preliminary data suggested that a higher sample concentration was not necessarily correlated with the reflection of more photons for each unique signature. However, note that the primary environmental factors affecting spectral collection include illumination intensity, sample turbidity, and relative sample concentration. Data for measured samples were imported into ENVI version 5.4 software (Harris Geospatial Solutions, Boulder, CO) for visual inspection. Areas called regions of interest (ROI) were selected for calculation of an average spectrum for greater analysis. The ROI selection criteria were that the region covered approximately $30 \%-40 \%$ of the visible sample and that the regions appeared uniform. Spectral and statistical data were then calculated for comparison and are described 
below. Using ENVI software, the ROI was defined in the image as shown in Figure 3. Using the Compute Statistics option, the statistics were calculated and are shown in Figure 4. The calculated statistics include pixel count and percentage per spectral band. Basic statistics such as minimum, maximum, mean, and standard deviation for each band were also generated. Histograms with frequency distributions (number of points, cumulative points, percent for each bin, and cumulative percentage for each digital number) were also produced.

Figure 3. Example region of interest (ROI) (inner green circle).

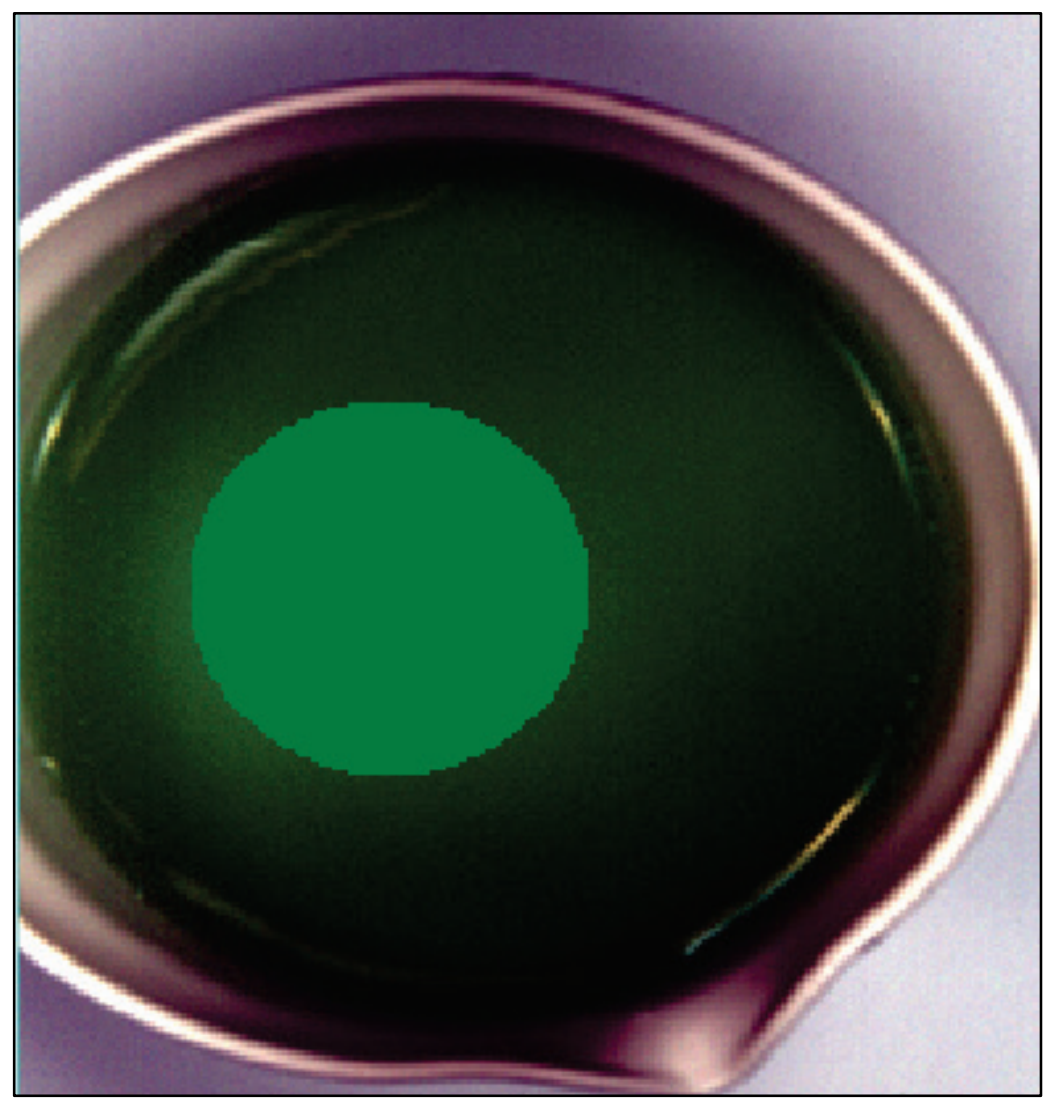


Figure 4. Image statistics generated in ENVI.

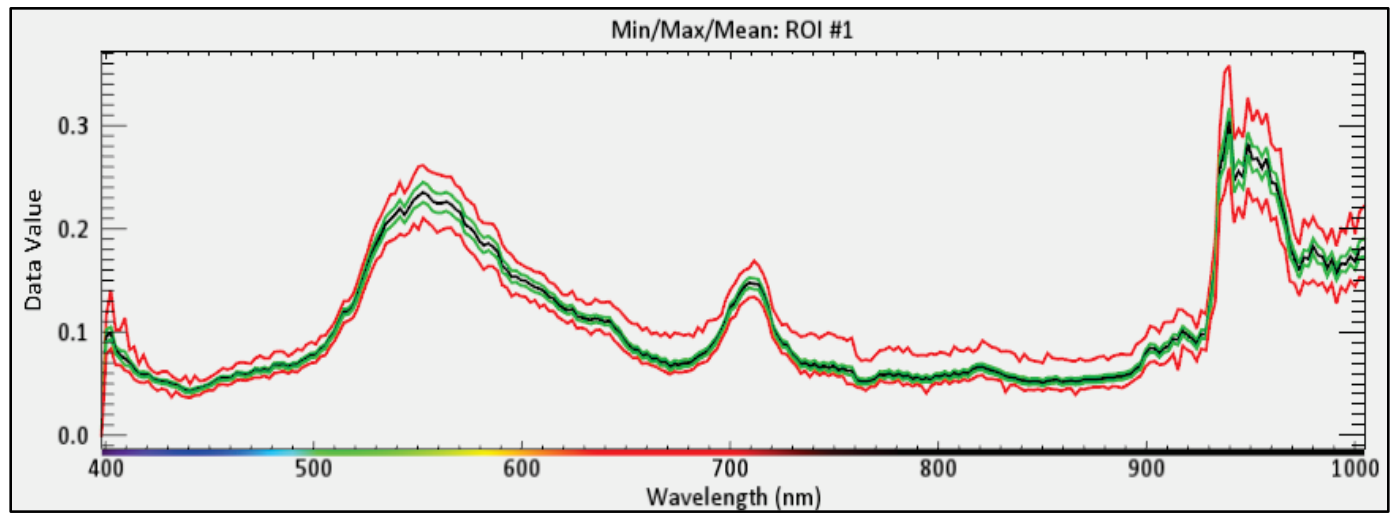

\begin{tabular}{|c|c|c|c|c|}
\hline ROI Summary & Pixel Count & & & \\
\hline $\mathrm{ROO} \# 1$ & 836 & & & \\
\hline \multicolumn{5}{|l|}{ ROI \#1 } \\
\hline Basic Stats & Min & $\operatorname{Max}$ & Mean & Stdneve \\
\hline Band 1 & 0.000000 & 0.000000 & 0.000000 & 0.000000 \\
\hline Band 2 & 0.078331 & 0.116058 & 0.095983 & 0.006251 \\
\hline Band 3 & 0.083666 & 0.140529 & 0.098814 & 0.006094 \\
\hline Band 4 & 0.068258 & 0.102173 & 0.082480 & 0.003806 \\
\hline Band 5 & 0.067939 & 0.102497 & 0.077917 & 0.004186 \\
\hline Band 6 & 0.062739 & 0.113750 & 0.074423 & 0.006667 \\
\hline Band 7 & 0.061257 & 0.082645 & 0.069948 & 0.002909 \\
\hline Band 8 & 0.054033 & 0.085674 & 0.064071 & 0.003363 \\
\hline Rand 9 & ก ก51 11.9 & 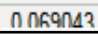 & ก ก59154 & ก กกวก15 \\
\hline
\end{tabular}

\subsection{Image analysis}

Initial laboratory data were acquired with a device designed for reflectance measurements. However, because the sample solution was transparent, it required a different analysis pipeline than a typical two-dimensional reflectance measurement. For example, a portion of the illumination undergoes reflection at the surface, but reflections continue down through the solution until the turbidity attenuates the impending light. In addition, reflections at any depth are attenuated by the solution on the return back through the sample. Spectral intensity data were extracted in the ENVI hypercube format and in comma separated value format for each ROI. BG-11 media was used as a blank to obtain a spectral baseline. From there, a rolling median filter was applied, centered over every three measurements using the freeware statistical program R v. 3.5.1 and R-Studio v. 1.1.383. Code for this can be found in the Appendix. 


\section{Results}

\subsection{Light scattering and cyanobacteria concentration}

By calculating the differences between increasing concentrations of sample spectra, the relationship between reflectivity and concentration could be explored. Using this method, a decrease in reflectance was noted with increasing concentration. This was attributed to the absorption of the reflected light with depth attenuated down the sample. Figure 5 shows the increasing concentrations of Anabaena sp. and M. aeruginosa cyanobacteria acquired with the Resonon hyperspectral camera.

Figure 5. Laboratory acquired samples of increasing concentrations of Anabaena (A) and Microcystis (B) cyanobacteria.

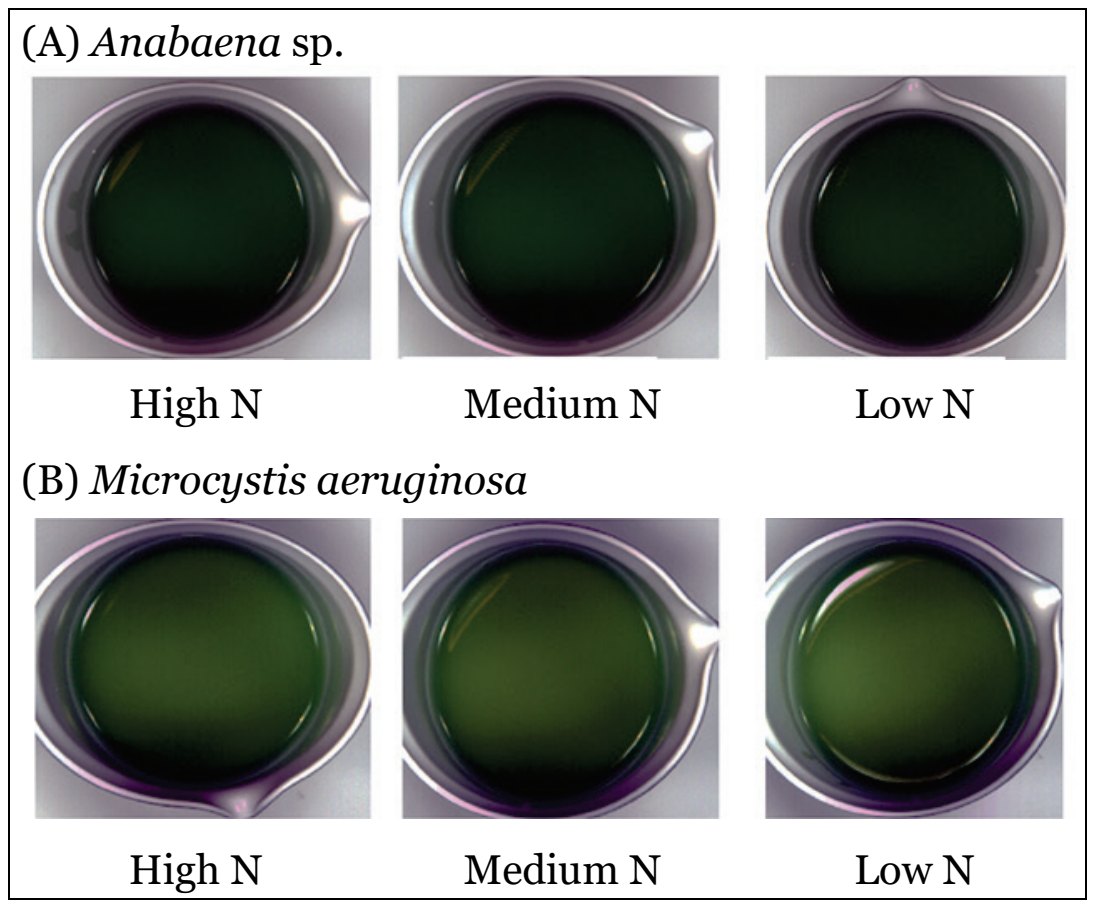

Increasing concentrations of the cyanobacteria in suspension increased the turbidity of the solution as determined by optical density (OD at $680 \mathrm{~nm}$ ) (Figure 6). This caused an increase in the scattering of the light as it was transmitted into the media, which coincided with decreased reflection and signal loss as the reflected light was transmitted back out through the sample. 
Figure 6. Spectral comparison of different concentrations/dilutions of Anabaena sp. both the raw spectrum $(A)$, instantaneous rate of change $(B)$.

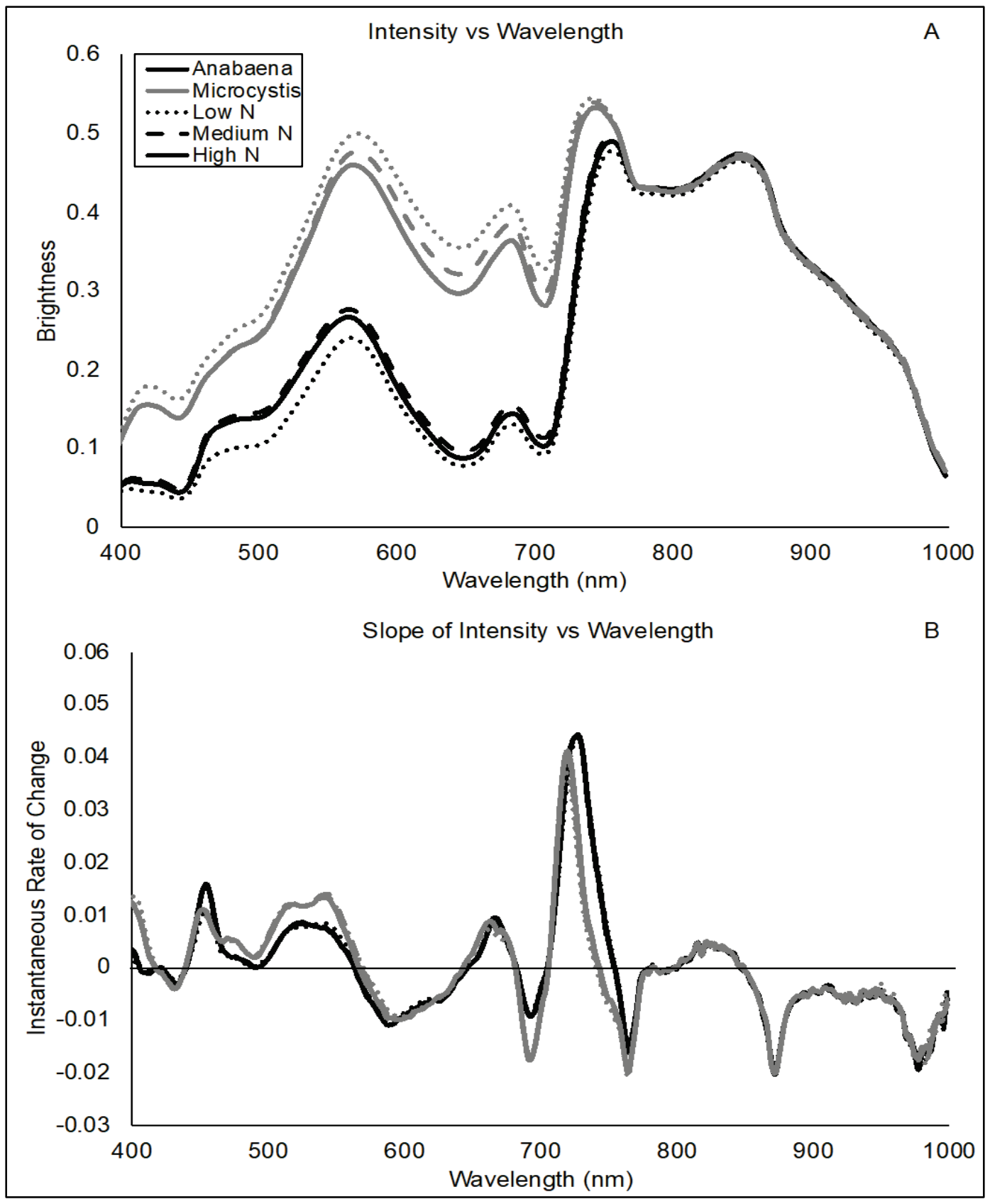

This technique is a combination of conventional absorption and reflection spectroscopy. Then intensity of light being reflected back to the detector is not a simple linear function. It can be seen from the analysis of the laboratory data in Figure 6 that increasing density is not indicative of more photons being reflected from the solution for each species unique spectral signature. 


\subsection{Cyanobacteria spectral comparisons}

Analysis of the laboratory results indicate sufficiently unique features in cyanobacteria spectra among genera to assist in biomass detection and identification. However, a comparison of these spectra with natural background levels and under-stressed conditions can provide information on the robustness of newly developed analysis algorithms, which is required for success in the field.

Figure 6 also demonstrates that the spectrum for two different genera of cyanobacteria (M. aeruginosa and Anabaena sp.), under replete culturing conditions, can be used to discriminate between genera using simple band ratios. While the basic shapes between the spectra are similar, inflection points occur at different wavelengths along with differences in slopes between spectra. Therefore, taking the first derivative of hyperspectral signatures may provide better separation between cyanobacteria genera of interest compared to multispectral and other imaging techniques.

Identification of anomalous regions by taking the first derivative of hyperspectral signatures may prove less computationally intensive when attempting to locate emerging bloom events. However, this is largely dependent on the spectral similarity between cyanobacteria and other algae of interest and the overall background characteristics of the lake or reservoir in question. Ideally, first derivative analysis may provide more efficient identification with less false positives when transitioning to the field. Analysis of field data will be required to make the determination of which space(s) are preferable to perform the analysis.

\subsection{Separating signatures from co-cultures of cyanobacteria}

The following is a definition of nomenclature for spectral imaging of laboratory results that were produced in a hypercube format. Each data set is defined as, $\mathbf{P} \mathbf{x}_{\lambda}$, where $\mathbf{X}$ is the dimensional spatial position vector for each layer of pixels in the set, and $\lambda$ is the layer in the stack for each set of wavelengths collected. Most of the area covered within $\mathbf{X}$ should contain background signal. Specifically, $\mathbf{X}$ is the physical coordinates of the spectrum measured, $\lambda$ is the wavelength, and $\mathbf{P} \mathbf{x}_{\lambda}$ is the actual reflectance at that position $\mathbf{X}$ at wavelength $\lambda$.

Once background parameters have been calculated, it is possible to establish a data set that contains only data values that fall within a certain 
proportion of the statistical uncertainty $\sigma_{\lambda \mathbf{P}}$, where $\mathrm{K}$ is a predetermined constant and $\sigma_{\lambda} \mathbf{P}$ is the standard deviation of the data set for a give band $\lambda$. The outlying regions can then be populated with data interpolated from nearest neighbors so that all values in that set are within the limits calculated by looking at the entire set acquired and creating a new hypercube, $\mathbf{P} \mathbf{x}_{\lambda \text { background. }}$

Subtracting the background interpolated data from the original measured

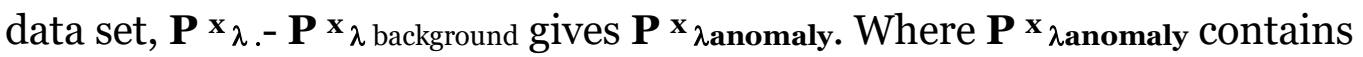
only regions that fall outside the background criteria and makes it possible to limit the analysis to only the anomalous regions contained in $\mathbf{P} \mathbf{x}_{\lambda}$. This reduces the comprehensive analysis to only the regions with spectral characteristics that fall outside of the baseline noise introduced by the background.

The calculation of $\mathrm{K} \sigma_{\lambda \mathbf{P}}$ determines the lower limit of detection concentration possible for the presence of a species contained in the reference library and quantification for any given data set.

\subsection{Future improvements}

Turbidity of the sample was directly associated with algal concentration; therefore, the measured reflectivity was not directly proportional to the concentration of the solution. Ultimately meaning that while this method may be good at determining relative abundances of phytoplankton populations, it likely will have challenges with determining total cell density. Developing a normalized spectrum for the detection of selected algae will provide baselines for comparison between species. Comparisons of the normalized spectra may indicate multi-band features that could then be used to identify algae using relative band ratios.

A more advanced method of analysis is to construct a reference library of spectra for the desired algae of interest. This reference library could be used as a set of baseline vectors to try to detect the presence of a cyanobacteria bloom through more complex analysis techniques. 


\section{Conclusions and Future Directions}

This report details how to perform hyperspectral imaging of cyanobacteria in the laboratory, how to process the images, and how to spectrally separate mixtures of algae.

Laboratory mono and mixed cultures have been inspected to determine if cyanobacteria have significantly unique hyperspectral properties orthogonal to each other to allow for early detection and monitoring in the field. Comparisons between cyanobacteria and non-harmful algal species will likely confer the greatest differences and provide optimal separation for identification; however, this was not conducted in this report.

Laboratory settings offer a controlled environment to study the spectral properties of various cyanobacteria; however, real-world conditions will influence absorption and reflectance characteristics. Complications from environmental conditions under which the imagery is collected make characterizing spectral properties more complicated than just looking for a particular spectral signature. The case of a pure spectrum representative of the algae of interest will be a rare case in nature, and in most instances, algae spectrum will have a partial component of a background (non-HAB) signature such as the lake or lake-bottom spectrum. In addition, spectra will almost certainly be influenced by the inherent properties of the water column (suspended sediment, dissolved compounds, or suspended organic matter, for example).

The earlier harmful cyanobacteria can be detected, the sooner management strategies can be implemented, thereby reducing the impact or avoiding the problem all together. By establishing and validating laboratory-based methods for isolating species-specific algal spectral signatures, this research lays the foundational groundwork for the development of low-cost sensors capable of remotely sensing cyanobacteria. Satellite- and airplane-based remote sensing has traditionally been used to map cyanobacteria blooms and can map cyanobacteria blooms over large spatial scales; however, these sensors can be expensive, are not highly resolute, tend to have low temporal collection frequency, require specialized processing methods, and, in the case of manned aircraft, increase personnel risk (Hunter et al. 2016). Recent advances in unmanned airborne, waterborne, and subsurface remotely operated systems as well as expanded federal guidelines provide an 
opportunity for the implementation of unmanned technology to be used in HAB monitoring and detection. The development of a low-cost sensor and processing methodologies capable of quickly identifying and quantifying the presence of cyanobacteria in the environment has been identified as a technological need for integration onto unmanned platforms to be used for early detection in the future (Kislik et al. 2018).

Methods developed in this laboratory research can be integrated into subsequent future experiments and adapted for field-based experiments that will have direct application to remotely sensed data collection, processing, and evaluation. Further research on cyanobacteria spectral properties in varying environmental and field conditions is needed. Field collection using UAV-based high-resolution hyperspectral data collected concurrently with point samples will be critical for determining spectral signatures of cyanobacteria in varying environments. Additionally, utilizing unmanned platforms in conjunction with existing traditional remotely sensed will establish best practices and address methodologies and use cases for UAV-based sensors in HAB detection and monitoring. Coupling the integration of inexpensive cyanobacteria sensors with advanced remotely operated semi-autonomous unmanned systems could provide a complementary solution for sampling large spatial scales with increased spectral resolution and temporal frequency at a lower cost. 


\section{References}

Brooks, B. W., J. M. Lazorchak, M, D. A. Howard, M-M. V. Johnson, S. L. Morton, D. A. K. Perkins, E. D. Reavie, G. I. Scott, S. A. Smith, and J. A. Steevens. 2015. “Are Harmful Algal Blooms Becoming the Greatest Inland Water Quality Threat to Public Health and Aquatic Ecosystems?" Environ Toxicol Chem 35: 6-13. doi: 10.1002/etc.3220

Hunter, P. D., M. W. Matthews, T. Kutser, and A. N. Tyler. 2016. "Remote Sensing of Cyanobacterial Blooms in Inland, Coastal, and Ocean Waters." Handbook of Cyanobacterial Monitoring and Cyanotoxin Analysis. John Wiley and Sons.

Kislik, C., I. Dronova, and M. Kelly. 2018. "UAVs in Support of Algal Bloom Research: A Review of Current Applications and Future Opportunities.” Drones 2(4): 35.

Mehrubeoglu, M., M. Teng, and P. Zimba. 2014. "Resolving Mixed Algal Species in Hyperspectral Images.” Sensors 14:1-21. doi: 10.3390/s140100001

Oppelt, N., F. Schulze, K. Doernhoefer, I. Eisenhardt, and I. Bartschl. 2012. "Hyperspectral Classification Approaches for Intertidal Macroalgae Habitat Mapping: A Case Study in Heligoland." Opt Eng 51: 111703-1-111703-11. doi: 10.1117/1.OE.51.11.111703

Reif, M. 2011. Remote Sensing for Inland Water Quality Monitoring: A US Army Corps of Engineers Perspective. ERDC/EL TR-11-13. Vicksburg, MS: US Army Engineer Research and Development Center.

Reif, M., C. Piercy, J. Jarvis, B. M. Sabol, C. Macon, R. Loyd, P. Colarusso, H. Dierssen, and J. Aitken. 2012. Ground Truth Sampling to Support Remote Sensing Research and Development: Submersed Aquatic Vegetation Species Discrimination Using an Airborne Hyperspectral/Lidar System. ERDC TN-DOER-E3O. Vicksburg, MS: US Army Engineer Research and Development Center.

Resonon, Inc. 2018. 123 Commercial Dr., Bozeman, MT.

Ross, C., L. Santiago-Vázquez, and V. Paul. 2006. “Toxin Release in Response to Oxidative Stress and Programmed Cell Death in the Cyanobacterium Microcystis aeruginosa." Aquatic Toxicology 78: 66-73. doi: 10.1016/j.aquatox.2006.02.007

Vincent R. K., X. Qin, R. M. L. McKay, J. Miner, K. Czaikowski, J. Savino, and T. Bridgeman. 2004. "Phycocyanin Detection from LANDSAT TM Data for Mapping Cyanobacterial Blooms in Lake Erie." Remote Sensing of Environment 89: 381392. doi: 10.1016/j.rse.2003.10.014 


\section{Appendix: R Script for Hyperspectral Data Analysis}

$\operatorname{library}(z o o)$

\#read in raw signatures

data1 <-

read.csv("/Users/rdel1klp/Documents/Project_Data/Hyperspectral/Grow th_Curves/csv-files/Anabaena-exp1-spectrum-05032017.csv")

data2 $<-$ rollmedian(data1,k=5, align="center")

write.csv(data2,"/Users/rdel1klp/Documents/Project_Data/Hyperspectra 1/Growth_Curves/csv-files/Anabaena-exp1-spectrum-05032017smoothed.csv")

\#\# \# cyano spectral comparisons

data1 <-

read.csv("/Users/rdel1klp/Documents/Project_Data/Hyperspectral/cyan o-comparison-spectra.csv")

data2 <- rollmedian(data1,k=3, align="center")

write.csv(data2,"/Users/rdel1klp/Documents/Project_Data/Hyperspectra l/cyano-comparison-spectra-smoothed.csv")

\#\#\# mixed spectral comparisons

data $1<-$

read.csv("/Users/rdel1klp/Documents/Project_Data/Hyperspectral/mixe d-spectra-06012017.csv")

data2 $<$ - rollmedian(data1,k=3, align="center")

write.csv(data2,"/Users/rdel1klp/Documents/Project_Data/Hyperspectra 1/mixed-spectra-06012017-smoothed.csv") 


\section{Unit Conversion Factors}

\begin{tabular}{|l|c|l|}
\hline Multiply & By & To Obtain \\
\hline microns & $1.0 \mathrm{E}-06$ & meters \\
\hline nanometer & $1.0 \mathrm{E}-09$ & meters \\
\hline milliliter & $1.0 \mathrm{E}-06$ & Liters \\
\hline
\end{tabular}




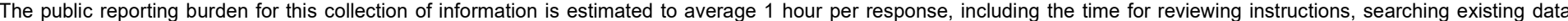

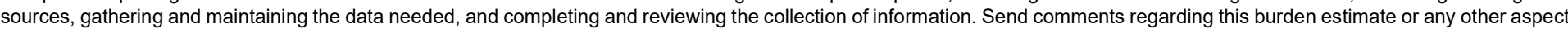

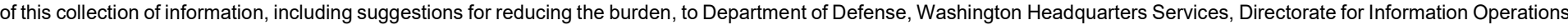

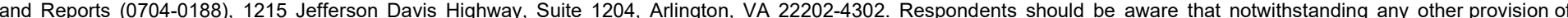
law, no person shall be subject to any penalty for failing to comply with a collection of information if it does not display a currently valid OMB control number. PLEASE DO NOT RETURN YOUR FORM TO THE ABOVE ADDRESS.

\begin{tabular}{l|l|l}
$\begin{array}{l}\text { 1. REPORT DATE } \\
\text { June } 2021\end{array}$ & $\begin{array}{l}\text { 2. REPORT TYPE } \\
\text { Final Report }\end{array}$ & 3. DATES COVERED (FrOm - To)
\end{tabular}

\section{TITLE AND SUBTITLE}

A Novel Laboratory Method for the Detection and Identification of Cyanobacteria Using

Hyperspectral Imaging:

Hyperspectral Imaging for Cyanobacteria Detection

5a. CONTRACT NUMBER

5b. GRANT NUMBER

5c. PROGRAM ELEMENT NUMBER

\section{AUTHOR(S)}

5d. PROJECT NUMBER

Kaytee Pokrzywinski, Cliff Morgan, Scott Bourne, Molly Reif, Kenneth Matheson, and Shea

Hammond

5e. TASK NUMBER

5f. WORK UNIT NUMBER

\section{PERFORMING ORGANIZATION NAME(S) AND ADDRESS(ES)}

Environmental Laboratory

US Army Engineer Research and Development Center

3909 Halls Ferry Rd

Vicksburg, MS 39180-6199

\section{SPONSORING/MONITORING AGENCY NAME(S) AND ADDRESS(ES)}

Aquatic Plant Control Research Program

US Army Engineer Research and Development Center

Vicksburg, MS 39180-6199

\section{PERFORMING ORGANIZATION REPORT NUMBER}

ERDC/EL TR-21-3

\section{SPONSOR/MONITOR'S ACRONYM(S)} APCRP

11. SPONSOR/MONITOR'S REPORT NUMBER(S)

\section{DISTRIBUTION/AVAILABILITY STATEMENT}

Approved for public release; distribution is unlimited.

\section{SUPPLEMENTARY NOTES}

Funding Account Code U4363405; AMSCO Code 070598

\section{ABSTRACT}

To assist US Army Corps of Engineers resource managers in monitoring for cyanobacteria bloom events, a laboratory method using hyperspectral imaging has been developed. This method enables the rapid detection of cyanobacteria in large volumes and has the potential to be transitioned to aerial platforms for field deployment. Prior to field data collection, validation of the technology in the laboratory using monocultures was needed. This report describes the development of the detection method using hyperspectral imaging and the stability/reliability of these signatures for identification purposes. Hyperspectral signatures of different cyanobacteria were compared to evaluate spectral deviations between genera to assess the feasibility of using this imaging method in the field. Algorithms were then developed to spectrally deconvolute mixtures of cyanobacteria to determine relative abundances of each species. Last, laboratory cultures of Microcystis aeruginosa and Anabaena sp. were subjected to varying macro (nitrate and phosphate) and micronutrient (iron and magnesium) stressors to establish the stability of signatures within each species. Based on the findings, hyperspectral imaging can be a valuable tool for the detection and monitoring of cyanobacteria. However, it should be used with caution and only during stages of active growth for accurate identification and limited interference owing to stress.

\section{SUBJECT TERMS}

Cyanobacteria, Cyanobacterial blooms-Detection, Hyperspectral imaging, Water quality-Monitoring

\section{SECURITY CLASSIFICATION OF:}

\begin{tabular}{|l|l|l|}
\hline a. REPORT & b. ABSTRACT & c. THIS PAGE \\
Unclassified & Unclassified & Unclassified \\
\hline
\end{tabular}

17. LIMITATION OF ABSTRACT

SAR
18. NUMBER

OF PAGES

26 19a. NAME OF RESPONSIBLE PERSON Kaytee Pokrzywinski

19b. TELEPHONE NUMBER (Include area code) 262-364-6556 